\title{
Errata
}

\section{A Correction to: "The Products in the Steenrod Rings of the Complex and Sympletic Cobordism Theories"}

(Vol. 10, No. 3, 1975, pp. 581-600)

By

Tetsuya AIKawA*

Page 582, line 8, "the main theorem" should be read as "Theorem 4.1".

Page 582, line 9, "I. Kojima" should be read as "I. Kozma".

* Department of Mathematics, Okayama University, Okayama. 
